\title{
Knowledge and awareness of human papillomavirus and intention with regard to human papillomavirus vaccine uptake by female tertiary students in the Eastern Cape province
}

\author{
A Chikandiwa, BE van Wyk
}

\author{
Admire Chikandiwa, MBChB, MPH, DipHIVMan, Researcher \\ Wits Reproductive Health and HIV Research Institute, University of the Witwatersrand \\ Brian Eduard van Wyk, MSc, DPhil, Associate Professor \\ School of Public Health, University of the Western Cape \\ E-mail: bvanwyk@uwc.ac.za \\ Keywords: human papillomavirus, awareness, knowledge, vaccine, health beliefs
}

\begin{abstract}
In 2008, two human papillomavirus (HPV) vaccines, Cervarix ${ }^{\circledR}$ and Gardasil ${ }^{\circledR}$, were licensed for use in South Africa. Initial models showed that vaccination could lead to an approximate $70 \%$ decline in cervical cancer cases. This paper describes the knowledge, awareness and health beliefs about HPV and the HPV vaccine, and the intentions of female tertiary students with respect of being vaccinated with the latter. An observational, descriptive and analytical cross-sectional survey was conducted among 150 female tertiary students at a university in the Eastern Cape province in this regard. Knowledge and awareness of HPV and the HPV vaccine were poor. Only $22.7 \%$ of the students were aware of HPV and that an HPV vaccine was available in South Africa. However, most respondents $(80 \%)$ reported a willingness to be vaccinated. Being aware of the existence of a Papanicolaou smear, higher knowledge of HPV, higher perceived vaccine effectiveness and higher perceived severity of HPV infection were significantly associated with increased willingness to be vaccinated. There is a need for education on HPV and its vaccination in South Africa. An effective vaccine marketing strategy should emphasise the effectiveness of the vaccine, the susceptibility of women to contracting HPV and the severity of being infected with the human immunodeficiency virus.
\end{abstract}

P Peer reviewed. (Submitted: 2013-02-27. Accepted: 2013-07-04.) ๑ SAJEI

South Afr J Epidemiol Infect 2013;28(4):215-220

\section{Introduction}

Women in South Africa have a high prevalence of human papillomavirus (HPV) infection. The highest rates of infection are found in sexually active adults who are younger than 25 years of age. ${ }^{1}$ HPV type 16 (high risk) is the most dominant type that is found in South Africa. ${ }^{2}$ Cervical cancer is the second most common cancer in South African women. There is an overall age-standardised incidence rate of 30/100 000/ year. ${ }^{3}$ After it became evident that HPV played a role in the genesis of cervical cancer, vaccines against HPV infections were developed. Currently, a massive drive for vaccination is underway worldwide. ${ }^{4,5}$ In March 2008, two new prophylactic HPV vaccines, a bivalent vaccine that targets HPV 16 and 18 (Cervarix ${ }^{\circledast}$, GlaxoSmithKline), and a quadrivalent vaccine directed at HPV 16, 18, 6 and 11 (Gardasi ${ }^{\circledR}$, Merck, and Sharpe and Dohme) were licensed for use in South Africa. However, they are not yet available in the public health sector. ${ }^{6}$ Modelling studies have shown that if fully implemented, vaccination can lead to a $31 \%$ reduction in precancerous lesions, which translates to an approximate $68 \%$ decline in cervical cancer cases. ${ }^{7}$ Therefore, a vaccination programme is an urgent priority in South Africa for the primary prevention of cervical cancer. This complements other strategies against cervical cancer that are already being implemented, such as the national cervical screening programme, aimed at the secondary prevention of cancer since not all women will be vaccinated. ${ }^{8}$

Research ${ }^{1,9}$ has found that between $10 \%$ and $39 \%$ of sexually active young adult women aged 18-25 years are infected with high-risk HPV at any point in time. Since the majority of university students are in that age group, they can be used as a proxy for this high-risk group. It is also noteworthy that female students will soon be mothers, if they are not already so, and are more likely to play a key role in HPV vaccination in the future if the universal vaccination of 11- to 12-yearold girls is recommended in South Africa, as has been the case in other countries. ${ }^{10}$ This is largely because people often infer others' needs from their own, and thus one may assume that women would deduce their daughter's need for HPV vaccination based on their own perceived needs. ${ }^{11}$

The health belief model (HBM) suggests that key predictors for the acceptability of any vaccine include perceived disease likelihood and severity, perceived vaccine benefits and barriers, and cues to action. ${ }^{12-14}$ In the context of HPV vaccination, "perceived likelihood" is the belief that HPV infection and cervical cancer are likely outcomes. "Perceived severity" is the belief that HPV infection and cervical cancer would have serious negative health consequences. "Perceived vaccine 
effectiveness", i.e. perceived benefit, is the belief that the HPV vaccine will reduce the risk of HPV infection or cervical cancer. Perceived barriers can be any perceived impediment to vaccination, such as cost. Cues to action are situational factors that prompt one to get vaccinated. Interventions ${ }^{15,16}$ guided by the HBM have been shown to increase vaccination rates.

A significant body of literature exists on issues relating to HPV vaccine acceptability. ${ }^{17}$ However, these studies focus on awareness and knowledge of cervical cancer, HPV and the HPV vaccine in relation to potential uptake and resistance in developed countries. To date, little research has been conducted in developing countries, and in South Africa, in particular. It is equally important that the predictors of women's interest in HPV education and their desire to have the HPV vaccination have not been characterised in this country where a significant number of females are able to benefit from the vaccination. Hence, it is necessary that local information guides the formulation of policies, and the introduction and propagation of the HPV vaccination in South Africa.

This paper describes the knowledge and awareness of HPV and the HPV vaccine in female university students, and factors that influenced their willingness to be vaccinated with the HPV vaccine.

\section{Method}

This was an observational, descriptive and analytical crosssectional study, conducted in 2009, in a university in the Eastern Cape province to describe the knowledge, awareness and health beliefs about HPV and the HPV vaccine, and factors that influenced the intentions of female students with regard to vaccine uptake.

\section{Study population and sampling}

Participants were female students registered at the university in 2009 and attending lectures at the time of the study ( $n=5000$ ). Based on a population of 5000 , using a very conservative estimate that $20 \%$ of them were aware of HPV and cervical cancer, and allowing $15 \%$ or $25 \%$ as the worst acceptable values using a 95\% confidence interval, the required calculated sample size was determined as 150 , using Epi Info ${ }^{\mathrm{TM}}$.

A stratified random sampling method was employed to obtain a representative sample of the female students. Each of the five faculties was considered to be a homogeneous stratum. $A$ random sample of 30 participants was then selected from each faculty. Stratified sampling was chosen mainly as a means of saving time and money because the target population was spread out.

\section{Ethical considerations}

Ethical clearance for the study was granted by the University of the Western Cape Senate Research Committee. Permission to conduct the study was obtained from the registrar of the university where the research was conducted. Participation in the study was voluntary and based on informed consent. Anonymity and confidentiality were maintained at all times.

\section{Data collection procedure}

Self-administered, anonymous, confidential questionnaires consisting of closed-ended questions were used to collect information. Interested participants in each faculty office were issued with an information sheet which they read and signed, before completing an attached questionnaire to demonstrate their informed consent. The information sheet explained the purpose of the survey and provided study participants with detailed information on their rights, as enshrined in the Human Sciences Research Council's ${ }^{18}$ ethical guidelines. At the end of each day, the five offices were visited and the completed questionnaires collected, until 30 were filled in each faculty. It took two-and-a-half weeks to obtain the required number (150) of completed questionnaires.

\section{The instrument}

The questionnaire (survey instrument) used in this study was a modified version of the knowledge and perceptions (KAP) survey. ${ }^{19}$ Questions to ascertain knowledge of the existence of the HPV vaccine, perceived barriers and benefits, self-efficacy with regard to obtaining more HPV-related education and the desire to receive the HPV vaccination, were incorporated in the KAP survey for the purpose of this study. Each HBM variable was operationalised via a 4-point Likert scale. In addition, questions on participants' number of sexual partners in the last 12 months, and their intentions with regard to obtaining more information on HPV and being vaccinated with the HPV vaccine to prevent HPV infection were added. Demographic characteristics included age, level of study at the university and ethnic background.

\section{Data analysis}

Epi Info ${ }^{\mathrm{TM}} 7$ was used for data analysis in the study. Double entry was carried out and both data sets compared using the Epi Info ${ }^{\text {TM }}$ data compare function. Any differences between the two data sets were checked and corrected appropriately using the original questionnaire.

Means and standard deviations (SD) were calculated for continuous variables for the descriptive analysis, and frequencies computed for categorical variables. Bivariate analysis was performed to assess the relationship between predictor variables and intention with regard to vaccine uptake.

\section{Results}

\section{Demographic and behavioural characteristics}

The mean age of the respondents was 22 years (SD 2.3 years). 
Most respondents were South African (70.7\%) and black (88\%). Most (79\%) were senior students, i.e. second-, thirdand fourth-year students, taking undergraduate degrees) (Table I).

Table I: The demographic and behavioural characteristics of the participants $(n=150)$

\begin{tabular}{|l|c|c|}
\hline Characteristics & Frequency & Percentage \\
\hline Age (years) & & \\
\hline $18-20$ & 37 & 24.7 \\
\hline $21-24$ & 87 & 58 \\
\hline $25-30$ & 26 & 17.3 \\
\hline Race & & \\
\hline Black & 132 & 88 \\
\hline Coloured & 8 & 5.3 \\
\hline Asian or Indian & 6 & 4 \\
\hline White & 4 & 2.7 \\
\hline Nationality & & \\
\hline South African & 106 & 70.7 \\
\hline Non-South African & 44 & 29.3 \\
\hline Year of study & & \\
\hline First & 31 & 20.7 \\
\hline Second & 66 & 4 \\
\hline Third & 107 & 22.7 \\
\hline Fourth & 40 & 8 \\
\hline Postgraduate & & 41.3 \\
\hline Behavioural characteristics & 12 & 37.4 \\
\hline In a relationship & 7 & \\
\hline Sexually active & & \\
\hline Had more than one sexual partner in the & & \\
\hline last six months & & \\
\hline Used a condom during the last sexual & & \\
\hline encounter & & \\
\hline
\end{tabular}

Most respondents reported being in a relationship $(71 \%)$ and sexually active $(71 \%)$. More than a third of sexually active active respondents $(37 \%)$ indicated that they had had more than one sexual partner in the past six months. More than half of the sexually active participants $(60.7 \%)$ indicated that they used a condom during their last sexual encounter.

\section{Knowledge and awareness of human papillomavirus and the human papillomavirus vaccine}

Few study participants $(23 \%)$ had heard of HPV, whereas most were familiar with cancer of the cervix $(90 \%)$ and the Papanicolaou (Pap) smear (70\%) (Table II). Media (electronic and print) was the most common source of information that reached $58 \%$ of respondents. Healthcare institutions ranked second $(25 \%)$, followed by family and friends $(25 \%)$.

Knowledge was generally limited. None of the participants were able to answer all 11 questions correctly. The mode score was 4 with a median of 4 . Less than $5 \%$ (seven) answered $75 \%$ of the questions correctly, and $22 \%$ answered none of them correctly. Most respondents $(72 \%)$ were "not sure" of
Table II: Knowledge and awareness of human papillomavirus and the human papillomavirus vaccine

\begin{tabular}{|l|c|c|}
\hline Knowledge and awareness & Frequency & Percentage \\
\hline Knowledge items & & \\
\hline HPV can cause herpes & 14 & 9.3 \\
\hline Genital warts are caused by HPV & 65 & 43.3 \\
\hline HPV can cause cancer of the cervix & 101 & 67.3 \\
\hline $\begin{array}{l}\text { If the Pap smear is normal, then you don't } \\
\text { have HPV }\end{array}$ & 21 & 14 \\
\hline $\begin{array}{l}\text { Changes in the Pap smear may indicate } \\
\text { that you have HPV }\end{array}$ & 43 & 28.7 \\
\hline $\begin{array}{l}\text { A negative test for HPV means that you } \\
\text { don't have HPV }\end{array}$ & 15 & 10 \\
\hline $\begin{array}{l}\text { Most people with HPV have no visible } \\
\text { signs or symptoms }\end{array}$ & 58 & 38.7 \\
\hline $\begin{array}{l}\text { I can transmit HPV, even if I don't have the } \\
\text { symptoms }\end{array}$ & 59 & 39.3 \\
\hline $\begin{array}{l}\text { Having one type of HPV means that you } \\
\text { can't acquire new types }\end{array}$ & 50 & 33.3 \\
\hline Awareness of & & \\
\hline HPV & & \\
\hline \begin{tabular}{l} 
Cancer of the cervix \\
\hline The Pap smear
\end{tabular} & 34 & 22.7 \\
\hline Source of information & 135 & 90 \\
\hline \begin{tabular}{l} 
Indicated at least one source \\
\hline Media
\end{tabular} & & 70.7 \\
\hline \begin{tabular}{l} 
Family and friends \\
\hline Healthcare providers
\end{tabular} & \\
\hline
\end{tabular}

HPV: human papillomavirus, Pap: Papanicolaou

the association between HPV and herpes. Only $9.3 \%$ gave the correct answer. On the other hand, $67.3 \%$ of respondents knew that there was a link between HPV and cancer of the cervix. Also, $43 \%$ of respondents were aware of the link between HPV and genital warts.

Less than a third $(32 \%)$ of respondents knew that a vaccine existed to prevent HPV and cervical cancer, and only $22 \%$ (33) of the study participants were aware that the vaccine was available in the country.

\section{Health beliefs and intention with regard to human papillomavirus vaccine uptake}

Table III indicates that risk perception by respondents of HPV was low. $41.3 \%$ believed that they were at risk. Most considered HPV infection and its consequences to be severe $(78 \%)$, and thought that being vaccinated would effectively protect them against HPV (83.3\%). Interestingly, most of the participants $(98 \%)$ indicated a desire to receive more information on the HPV infection, cervical cancer and the HPV vaccine.

Slightly over $80 \%$ of the students were willing to be vaccinated if the vaccine was available. Vaccine uptake would be greatest in study respondents if it could prevent cancer $(71.3 \%)$ and 
Table III: Health beliefs and intention with regard to human papillomavirus vaccine uptake

\begin{tabular}{|l|c|c|}
\hline $\begin{array}{l}\text { Health beliefs and intention with regard } \\
\text { to human papillomavirus vaccine } \\
\text { uptake }\end{array}$ & Frequency & Percentage \\
\hline Health beliefs & & \\
\hline Believe they are at risk of HPV infection & 62 & 41.3 \\
\hline Believe that HPV infection is severe & 117 & 78.8 \\
\hline Believe that the HPV vaccine is effective & 125 & 83.3 \\
\hline Want to be educated more on HPV & 147 & 98 \\
\hline Would opt to be vaccinated if & & \\
\hline It was available to them & 122 & 81.3 \\
\hline It prevented warts only & 64 & 42.7 \\
\hline It prevented cancer only & 107 & 71.3 \\
\hline It prevented both & 140 & 93.3 \\
\hline It was one dose, given once off & 106 & 70.7 \\
\hline It was two doses, given three months & 42 & 28 \\
\hline apart & & 117 \\
\hline The vaccine was free of charge & 64 & 78 \\
\hline The vaccine cost R500 & 126 & 82.7 \\
\hline It was recommended by a doctor & 88 & 58.7 \\
\hline It was recommended by a spouse, family \\
member or friend
\end{tabular}

HPV: human papillomavirus

both cancer and warts (93.3\%). There was low interest $(42.7 \%)$ in the vaccine if it could only prevent warts.

More clients (70\%) preferred to be vaccinated with a once-off dose, rather than two doses over a three-month period (28\%). Similarly, most participants (78\%) would opt for a vaccine if it was administered free of charge rather than having to assume the cost of R500 (42.7\%).

Table IV: Predictors of the uptake of the human papillomavirus vaccine

\begin{tabular}{|l|c|c|}
\hline Predictors & Odds ratio & $\begin{array}{c}95 \% \text { confidence } \\
\text { interval }\end{array}$ \\
\hline \multicolumn{2}{|l|}{ Sexual behaviour } & \multicolumn{2}{|l|}{} \\
\hline $\begin{array}{l}\text { Being in a relationship } \\
\text { Being sexually active }\end{array}$ & 0.64 & $0.94-5.16$ \\
\hline $\begin{array}{l}\text { Having > 1 partner in a six-month } \\
\text { period }\end{array}$ & 0.68 & $0.29-1.72$ \\
\hline $\begin{array}{l}\text { Used a condom during the last } \\
\text { sexual encounter }\end{array}$ & 1.04 \\
\hline Awareness and knowledge of HPV & & $0.41-2.61$ \\
\hline Awareness of cancer of the cervix & 2.43 & $0.76-7.79$ \\
\hline Awareness of HPV & 1.96 & $0.63-6.09$ \\
\hline Awareness of the Pap smear & 3.54 & $1.51-8.27$ \\
\hline Higher knowledge of HPV & 2.94 & $1.23-7.02$ \\
\hline Health beliefs & & \\
\hline $\begin{array}{l}\text { Believed themselves to be at risk of } \\
\text { HPV infection }\end{array}$ & 1.99 & $0.81-5.16$ \\
\hline The HPV infection can be severe & 5.42 & $2.23-13.2$ \\
\hline The HPV vaccine is effective & 10.09 & $3.84-16.5$ \\
\hline
\end{tabular}

Pap: Papanicolaou
A doctor's recommendation of the vaccine would result in higher potential vaccine uptake (84\%), although many participants indicated that a recommendation from a spouse, family member or friend would also motivate them to be vaccinated (58.7\%).

Table IV shows that the bivariate analysis found that being aware of the existence of a Pap smear, and having more knowledge about HPV, were associated with a fourfold [odds ratio (OR) of $3.54,95 \%$ confidence interval $(\mathrm{Cl}): 1.51-8.27)]$, and a threefold (OR of $2.94,95 \% \mathrm{Cl}: 1.23-7.02)$ increase in the odds of being willing to be vaccinated, respectively. Those who believed HPV and its consequences to be severe were observed to be five times (OR of $5.42,95 \% \mathrm{Cl}$ : 2.23-13.2) more willing to be vaccinated. Individuals who believed that the vaccine was effective were 10 times (OR of $10.09,95 \%$ $\mathrm{Cl}$ : 3.84-16.5) more willing to be vaccinated, compared to those who did not believe that the vaccine was effective.

\section{Discussion}

In this study, $22.7 \%$ of the participants were aware of HPV. This compares fairly well with findings from other studies. A systematic review of 60 studies ${ }^{17}$ on awareness of HPV among females of diverse backgrounds reported a range of between $13 \%$ and $93 \%$. In contrast to the low awareness of HPV in respondents in this study, most $(90 \%)$ were aware of cancer of the cervix. This could be because HPV is a microbiological term, and could be difficult for people without medical training people to recall, compared to the term "cancer of the cervix", which the general population would tend to recall more easily.

The study showed that $70 \%$ of the respondents had heard of the Pap smear. This is less than the $95 \%$ reported for females staying in an urban district in Cape Town. ${ }^{8}$ The difference could have arisen because the Cape Town study focused on women attending gynecological clinics and they could have been more aware of Pap smears because of their contact with associated health workers. In this study, it was worrying to note that more participants $(58 \%)$ cited media as a primary source of information, rather than healthcare workers $(25 \%)$. Similar findings were also reported [81\% (media) versus $2 \%$ (healthcare workers)] in a study by Moodley et al. ${ }^{8}$

However, it was encouraging that $67.3 \%$ of the students knew of an association between HPV and cancer of the cervix. This finding was almost the same $(68 \%)$ as the one that was reported in a study on female university students at Walter Sisulu University (formerly the University of Transkei). ${ }^{20}$

This study highlighted confusion between HPV and other sexually transmitted infections. For instance, only $9.3 \%$ of students knew that HPV did not cause herpes. A similar finding was also observed in a study ${ }^{21}$ on female university students, wherein $80 \%$ thought that there was a link between HPV and herpes genitalis.

Less than a third of respondents were aware of a vaccine to 
prevent HPV and cervical cancer, and even less (22\%) knew that the vaccine is available in the country. Eighty per cent of respondents would opt to be vaccinated if it was available to them, which confirms a high level of acceptability which has also been documented in the literature. In urban Turkey, acceptability of the HPV vaccine was even higher (96\%) in a nonrepresentative sample of women attending a gynecological clinic. $^{22}$ Locally, a qualitative study in the Western Cape province $^{6}$ on members of the community revealed that the need for a vaccine to prevent cancer resonated with the respondents, despite overall poor knowledge of cancer of the cervix and its causal relationship with HPV.

A great proportion of our study participants wanted a vaccine that would prevent both genital warts and cervical cancer. This finding confirmed what was reported by Hoover et al, i.e. that $97 \%$ of respondents would opt for a vaccine that could prevent both diseases.

This study also revealed that more clients $(70 \%)$ would prefer to be vaccinated with a once-off dose, rather than two doses over a three-month period (28\%). This may have implications with regard to the acceptability of the current vaccine which is to be administered via three doses, three months apart, with each dose costing R450. Therefore, the total cost of the vaccination would be $\mathrm{R} 1350 .{ }^{23}$ Moreover, the observation that a greater percentage of study participants $(78 \%)$ would prefer a vaccine that was administered free of charge rather than one costing R500 (42.7\%). This suggests that efforts should be invested in finding ways to make the vaccine more affordable, and hence accessible, to a greater proportion of the populace.

It was found that a doctor's recommendation for the vaccine would result in a potential $84 \%$ vaccine uptake, while a recommendation from a spouse, friend or family member would lead to an approximate $60 \%$ uptake. Such findings have been confirmed in studies that were carried out elsewhere..$^{24} \mathrm{~A}$ physician's endorsement of a vaccine has been identified as an important catalyst for vaccine acceptance. A number of studies ${ }^{25-27}$ have reported that higher acceptability of the vaccine was found in subjects who thought that their doctors would recommend it. This finding lends support to the notion that of various possible modes of action, physician recommendation is likely to be key to the success of HPV vaccination programmes. This is because physicians may be uniquely persuasive in addressing perceived barriers, for example, by initiating conversations with patients about their concerns, clarifying any misunderstandings and recommending the vaccine.

\section{Health belief model constructs as predictors of vaccine uptake}

HBM constructs have been reported to be predictors of potential vaccine uptake..$^{14,12} \mathrm{~A}$ number of studies ${ }^{25,28,29}$ have documented that a higher perceived risk of HPV infection was associated with higher vaccine acceptability. However, in this study, it was found that a higher perceived risk of HPV infection was not statistically significantly associated with higher vaccine acceptability.

The study also found that perceived severity was a significant predictor of willingness to be vaccinated. This finding is in contradiction which the documented literature, ${ }^{25-27}$ which states that perceived severity did not relate to vaccine acceptability. This difference could have arisen because these studies were carried out in a Western setting, whose population's beliefs could potentially differ from those of the predominantly African group in our study.

Perceived vaccine effectiveness was associated with greater willingness to be vaccinated in our study. This confirms the findings of previous studies. ${ }^{26,30}$ It was interesting to note that our study confirmed the literature ${ }^{24}$ reports that advocated vaccine effectiveness as the most important attribute of an acceptable vaccine.

\section{Limitations of the study}

Because of the study design, students with a lower frequency of accessing their e-mails were likely to be under-represented. Thus, the study may be subject to selection bias. Voluntary participation may also have resulted in students with an interest in the topic self-selecting for the study. However, since general knowledge of HPV was low in the respondents, it is unlikely that this was the case.

\section{Conclusion}

Considerable work needs to be carried out on educating the general population about HPV, cancer of the cervix and the HPV vaccine in view of the poor knowledge demonstrated by university students. It is perhaps assumed that university students should have better knowledge of these subjects than ordinary women in the community because of their potential to attain a higher education and social status. Healthcare institutions and workers need to focus on providing this information to women.

There is an urgent need to review the cost of the vaccine, or/ and to make it available in the public health sector so that it will be accessible to a greater number of clients. Vaccine marketing strategies could educate women on HPV, emphasise their susceptibility and the severity of the consequences, and convince them about the vaccine's effectiveness in preventing HPV and cervical cancer.

\section{References}

1. Alan B, Hoffman M, Cooper D, et al. Prevalence of high-risk papillomavirus in cervical specimens of women from Cape Town. Brazil: $19^{\text {th }}$ International Papillomavirus Conference; 2001.

2. Williamson A, Passmore J, Marais D, Rybicki E. HPV infection in southern Africa: prevalence, immunity and vaccine prospects. Life. 2002;53(4-5):253-258.

3. Mqogi N, Kellet P, Sitas F, Musa J. Incidence of histologically diagnosed cancer in South Africa 1998-1999. Johannesburg: National Cancer Registry of South Africa; 2004.

4. Wong PL. Young multiethnic women's attitudes toward the HPV vaccine and vaccination. Int 
J Gynecol Obstetr. 2008;103(2):131-135

5. World Health Organizatio. Report of the consultation on human papillomavirus vaccines Geneva: World Health Organization; 2005.

6. Harries J, Moodley J, Barone MA, et al. Preparing for HPV vaccination in South Africa: key challenges and opinions. Vaccine. 2008;27(1):38-44.

7. Alliance for Cervical Cancer Prevention. Natural history of cervical cancer: even infrequent screening of older women saves lives. New York: Alliance for Cervical Cancer Prevention 2003.

8. Moodley J, Kawonga M, Bradley J, Hoffman M. Challenges in implementing a cervica screening program in South Africa. Cancer Detection and Prevention. 2006;30(4):361-368.

9. Winer RL, Lee S, Hughes JP, et al. Genital human papillomavirus infection: incidence and risk factors in a cohort of female university students. Am J Epidemiol. 2003;157(3):218226.

10. Markowitz LE, Dunne EF, Saraiya M. Quadrivalent human papillomavirus vaccine: recommendations of the Advisory Committee on Immunization Practices (ACIP). MMWR Recomm Rep. 2007;56(RR-2):1-24

11. Serpell L, Green J. Parental decision-making in childhood vaccination. Vaccine. 2006;24(19):4041-4046.

12. Brewer NT, Weinstein ND, Cuit CL, Herrington JE. Risk perceptions and their relation to risk behavior. Annal Behav Med. 2004;27(2):125-130

13. Chapman GB, Coups EJ. Predictors of influenza vaccine acceptance among healthy adults. Prev Med. 1999;29(4):249-262.

14. Becker M. The Health Belief Model and personal health behavior. Thorofare: Slack; 1974.

15. Hawe P, McKenzie N, Scurry R. Randomized controlled trial of the use of a modified postal reminder card on the uptake of measles vaccination. Arch Dis Child. 1998;79(2):136.

16. Larson EB, Bergman J, Heidrich $F$, et al. Do postcards improve influenza vaccination compliance? Med Care. 1972;20(6):639-648.

17. Klug SJ, Hukelmann M, Blettner M. Knowledge about Infection with Human Papillomavirus: A Systematic Review. Prevent Med. 2008;46(2):87-98
18. Code of research ethics of the Human Sciences Research Council. Human Sciences Research Council [homepage on the Internet]. 2009. c2009. Available: http://www.hsrc. ac.za/about/researchEthics

19. McPartland T, Weaver S, Koutsky L. Men's perceptions and knowledge of human papillomavirus (HPV) infection and cervical cancer. J Am Coll Health. 2005;53(5):225-230.

20. Buga GAB. Cervical cancer awareness and risk factors among female university students. East Afr Med . 1998;75(7):411-416.

21. Yacobi E, Tennant C, Ferrante J, et al. University students' knowledge and awareness of HPV. Prev Med. 1999;28(6):535-541.

22. Baykal C, Al A, Ugur MG. Knowledge and interest of Turkish women about cervical cancer and HPV vaccine. Eur J Gynecol Oncol. 2008;29(1):76-79

23. GlaxoSmithKline to reduce price of HPV vaccine cervarix in South Africa. Medica News Today [homepage on the Internet]. 2008. c2010. Available from: http://www. medicalnewstoday.com/articles/131918.php

24. Zimet GD, Mays RM, Sturm LA, et al. Parental attitudes about sexually transmitted infection vaccination for their adolescent children. Arch Pediatr Adolesc Med. 2005;159(2):132-137.

25. Boehner CW, Howe SR, Bernstein DI, Rosenthal SL. Viral sexually transmitted disease vaccine acceptability among college students. Sex Transm Dis. 2003;30(10):774-778.

26. Dempsey AF, Zimet GD, Davis RL, Koutsky L. Factors that are associated with parental acceptance of human papillomavirus vaccines: a randomized intervention study of written information about HPV. Pediatrics. 2006;117(5):1486-1493.

27. Kahn JA, Rosenthal SL, Hamann T, Bernstein DI. Attitudes about human papillomavirus vaccine in young women. Int J STD AIDS. 2003;14(5):300-306

28. Friedman AL, Shepeard H. Exploring the knowledge, attitudes, beliefs, and communication preferences of the general public regarding HPV: findings from CDC Focus Group Research and implications for practice. Health Educ Behav. 2007;34(3):471-485.

29. Olshen $E$, Woods $E R$, Austin $S B$, et al. Parental acceptance of the human papillomavirus vaccine. J Adolesc Health. 2005;37(3):248-251.

30. Davis K, Dickman ED, Ferris D, Dias JK. Human papillomavirus vaccine acceptability among parents of 10- to 15-year-old adolescents. J Low Genit Tract Dis. 2004;8(3):188-194. 